\title{
Exploring a Feedback-Oriented Design Process Through Curved Folding
}

\author{
Emrecan Gulay \\ Aalto University \\ Espoo, Finland \\ emrecan.gulay@aalto.fi
}

\author{
Toni Kotnik \\ Aalto University \\ Espoo, Finland \\ toni.kotnik@aalto.fi
}

\author{
Andrés Lucero \\ Aalto University \\ Espoo, Finland \\ lucero@acm.org
}

\begin{abstract}
The advancement of computational design and fabrication technologies has allowed combining physical and digital processes in architecture. Existing methods for physical-digital integration offer limited support for explorations with folded non-linear surfaces. This paper introduces a feedback-oriented design approach linking physical models with digital tools to enhance ideation processes in architecture. We employ paper as a medium for translating simple mock-up ideas to more elaborate digital design models. We explain the physical exploration, 3D scanning, digital simulation, and fabrication processes. Then, we discuss the results, observations, and limitations of this design approach.
\end{abstract}

\section{CCS CONCEPTS}

- Human-centered computing $\rightarrow$ Interaction design theory, concepts and paradigms.

\section{KEYWORDS}

computer-aided design, physical models, ideation, design exploration

\section{ACM Reference Format:}

Emrecan Gulay, Toni Kotnik, and Andrés Lucero. 2021. Exploring a FeedbackOriented Design Process Through Curved Folding. In CHI Conference on Human Factors in Computing Systems (CHI '21), May 8-13, 2021, Yokohama, Japan. ACM, New York, NY, USA, 8 pages. https://doi.org/10.1145/3411764. 3445639

\section{INTRODUCTION}

Paper folding is a quick and effective manual technique for modeling three-dimensional (3D) volumes. Beginning with the invention of paper in Asia, the soft and malleable nature of sheet materials led to remarkable form exploration techniques (e.g., origami) $[28,29]$. During the 1920 s paper folding was taught as a course in the Bauhaus school of design by Josef Albers in the context of architectural pedagogy [22, 42]. The concept of folding has been comprehensively explored in architecture for the design of complex structural morphologies [10, 32]. From the beginning of the twentieth century, architects experimented with folded forms to achieve more height with better rigidity and to reduce the net weight of

\section{(c) (i) (2)}

This work is licensed under a Creative Commons Attribution-Share Alike International 4.0 License.

CHI '21, May 8-13, 2021, Yokohama, Japan

(c) 2021 Copyright held by the owner/author(s).

ACM ISBN 978-1-4503-8096-6/21/05.

https://doi.org/10.1145/3411764.3445639 structural components [40]. Folding allows architects to understand the physical constraints of their design ideas by manually deforming sheet materials. In recent years, there has been a growing interest in free-form architectural surfaces with the advancements in contemporary digital design and manufacturing methods [22]. Challenging construction procedures of such forms require an integration of physical and digital design processes from the early design stages. As Terzidis [45] points out, the technological transformation in architecture generated a need to integrate computerized models with manual design techniques.

With the influence of makers' movement [8] and significant advances in digital fabrication and computer-aided design technologies, touch-based design knowledge and physical model making are coming into prominence within both architecture and HCI communities [16]. Although prior research offers innovative ways of combining physical and digital processes, there are limitations to non-linear design exploration. Hence, there is room for testing with a specific focus on the feedback exchange between physical and digital design workflows to facilitate complex curved form explorations in the initial design processes [16]. By using the term workflow, we refer to the iterative progression of the design process from initiation to conclusion. Our work aims to expand the current understanding of digital design methods and support creative processes in the initial stages of architectural design. We study curved folding as a test case to combine physical and digital design workflows in the conceptual design process. Compared to polyhedral surfaces, curved folded paper surfaces can provide " $a$ richer yet still tractable class of surfaces for computer-aided design and computer graphics applications." [18] Due to their complex kinematics, we focused on curved folded paper models to study how physical and digital design workflows can inform one another for creating nonlinear forms. We are seeking answers to the following research questions:

1) How can we utilize simple mock-up models for translating initial design ideas to more elaborate digital design models?

2) How can we facilitate intuitive non-linear explorations by extracting feedback from physical and digital design processes?

The paper is structured as follows. First, we introduce an overview of relevant research within the fields of $\mathrm{HCI}$ and architecture. The following section explains our methodology and how the design process was implemented. Next, the design process is elaborated under four subsections. Finally, we discuss our results, observations, and limitations encountered during each design process. Lastly, we provide an overview of our main sections and illustrate future approaches to extend our research. 


\section{RELATED WORK}

The integration of physical and digital design platforms is a broad research area covering a variety of approaches [16] (e.g., digital fabrication, automated, hybrid, and tangible approaches). We have identified relevant related work that looks at the transition from digital to physical (i.e., TUIs and tabletops), and from physical to digital (i.e., curved folded models and 3D scanners).

\subsection{From Digital to Physical: TUIs and Tabletops}

Existing research in the HCI domain focuses on the transition between physical artifacts and virtual models in the context of tangible approaches. For example, the physical proxy method introduced by Sheng et al. [41] allows the detection of physical hand motions in real-time to manipulate virtual 3D models. In this method, sponge and clay materials are the primary physical manipulation instruments. Similarly, clay and sand materials $[19,37]$ have been used as a medium to address the "disjunction" between physical and digital through tangible user interfaces (TUI). Tangible transition methods $[3,48]$ have the potential to facilitate volumetric exploration in the initial architectural design process. However, material restrictions and confined interfaces limit the applicability of such techniques for architectural form exploration.

Another approach that has been used is interactive tabletops that translate physical information into the digital platform. For instance, Pictionaire [17] is an interactive tabletop system developed for design teams to transfer sketches from physical to a digital platform. The system is limited to two-dimensional (2D) imagery, and therefore it does not support three-dimensional (3D) artifacts. Some research implemented actuated interactive surfaces for the exploration of 3D non-linear geometries [30, 31, 39]. Geomotion Screen [34] is an example that supports the physical manipulation of virtual models through an actuated interactive surface. The realtime visual and haptic feedback allows users to create non-linear surfaces. Although actuated surfaces enable tectonic explorations, they may limit the creation of more complex curved surfaces (e.g., folded, twisted, etc.).

In addition to TUIs and tabletops, prior research explored diverse methods for integrating digital and physical realms. Willis et al. [50] introduce an interactive fabrication approach through a series of prototypes that transform touch and voice inputs into physical objects. Shapetape [5] is a bend and twist sensitive strip allowing users to directly manipulate the shape of a virtual curved geometry as a tangible tool. SPATA [47] tools combine physical measurement data with virtual design environments (i.e., $\mathrm{mCAD}$, mesh-based modeling, and 2D design.) As a real-time approach, the ModelCraft framework [44] translates physical geometric data into digital platforms through freehand annotations. Similarly, ShapeMe [49] detects the physical transformation of an object and links it in real-time with the virtual model. CopyCAD [11] provides projected feedback directly on a CNC milling machine by gathering information from physical objects. These related works intuitively combine digital and physical design, optimization, and fabrication methods to enhance various design processes.

\subsection{From Physical to Digital: Curved Folded Models and 3D Scanners}

The aforementioned tangible methods propose a fluent transition between physical and digital design workflows to enhance the design process. However, design platforms introduced in these methods do not offer the same flexibility a paper model affords. Curved paper folding is one of the remarkable and challenging test cases that provides a tangible representation of complex curved surfaces [28]. Curved folded models have been previously studied and implemented to support design processes. Huffman [18] established the current understanding of curved folded surfaces and studied ways to implement them for computational processes. Koschitz [23] proposed design approaches to apply curved folded geometries by distilling the works of artists, designers, and geometers. Supporting the design and reconstruction of curved folded surfaces has investigated through digital frameworks [9, 21]. An alternative way of using the curved paper folding technique was by employing scoring and manual folding strategies in the initial design stages [6, 7]. Chandra et al. [9] identified that "a significant obstacle in working with curved-folded geometries is the lack of appropriate computational tools in commercial CAD software for describing such geometries." Therefore, computing curved-folded surfaces can be particularly difficult due to their complex kinematic characteristics. Recent advancements in volumetric 3D scanning technologies made it possible to collect high-precision physical data from complex curved surfaces.

3D scanning is a physical to digital translation method that has the potential to provide 3D physical data without the need for intricate modeling procedures [26]. The 3D scanning approach has been utilized among architects and researchers to translate complex curved surfaces into digital platforms. During the 1990s, renowned architect Frank O. Gehry's office has used 3D scanners to digitize non-linear physical models [10]. Gehry explored his initial ideas through physical mock-up models alongside sketches, then traced them into digital models. His creative approach significantly contributed to the development of contemporary architecture. 3D scanning has been used as a tool to translate conceptual physical models to digital models by linking digital and non-digital media through a hybrid design process [35]. Laing et al. [27] employed laser scanning technology to integrate 3D scanned data into Building Information Modelling (BIM) software. With this technique, they were able to obtain highly accurate physical data of freeform surfaces. Various other methods have been suggested for computing and improving the accuracy of complex curved surfaces (e.g., via a rotary scanning configuration) [46] in the design and engineering fields [26].

\section{METHODOLOGY AND IMPLEMENTATION}

This research presents a feedback-oriented design approach that alternates between physical and digital modes of design. Therefore, a 1:1 scale structural system's form and performative capabilities unfold from feedback cycles between physical and digital processes.

As Koskinen [24] states, "when researchers construct something, they discover things that would otherwise go unnoticed." We adopted a research through design $(\mathrm{RtD})$ approach $[12,24,53]$ and the material engagement framework [38] as a basis to generate a design method 


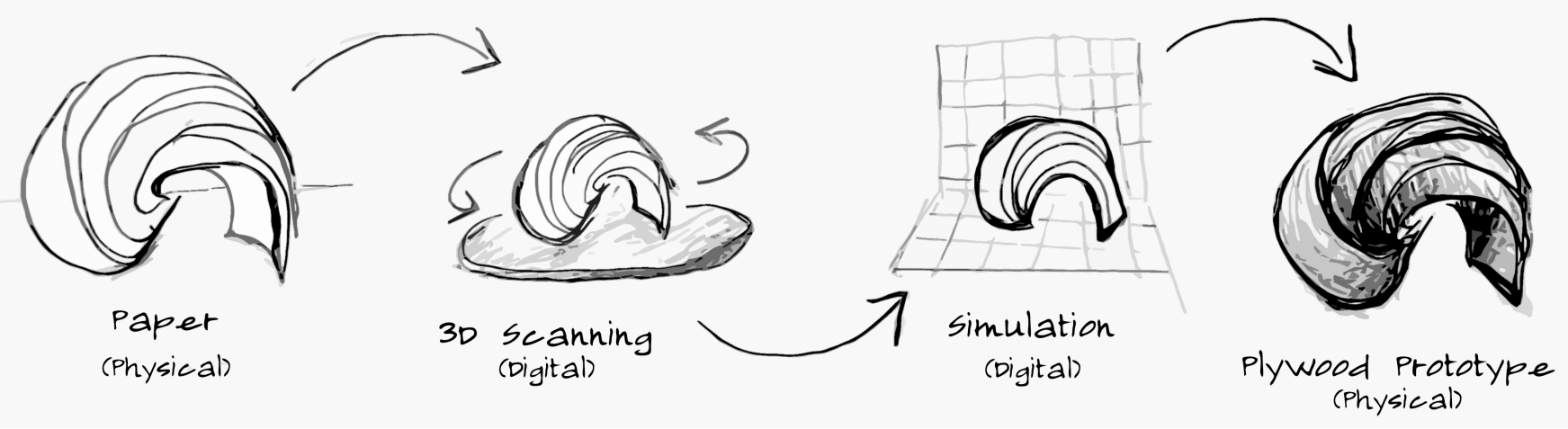

Figure 1: The feedback-oriented design process employs the curved folding technique as a test case to integrate physical and digital workflows of architectural design. The design process incorporates physical exploration, 3D scanning, digital simulation, and fabrication processes.

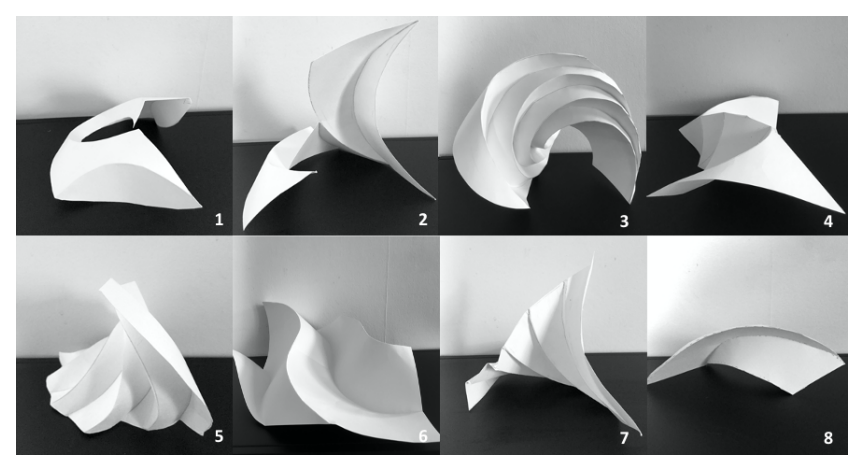

Figure 2: Volumetric exploration by changing the physical form of a single sheet of A3 paper. Form finding process and examples of curved folded mechanisms.

that unites digital and non-digital mediums to facilitate spatial exploration. We investigate the design process by documenting and reflecting on physical and digital models. When each physical and digital design process subsequently informs one another, they also generate knowledge and present concrete examples that can be utilized by practicing designers and researchers within the domains of HCI and architecture (Fig.1) [53].

The scope of our investigation is the preliminary design exploration phase. We employ a manual curved folding strategy as a testing case to study the feedback exchange between physical and digital platforms. The folding process follows a bottom-up approach [23] in which the logics of curved fold lines and rulings drive the design process. Thus, changing the physical properties of a single sheet of paper through variations of fold lines guides the design outcome of curved folded models (Fig.2). The primary design objective is to create rigid curved folded volumes that can be traced and transformed into digital 3D models for testing and fabrication.

The design process was mainly carried out by the first author of the paper, who has training and work experience in architecture. As a starting point of this design process, we determined three

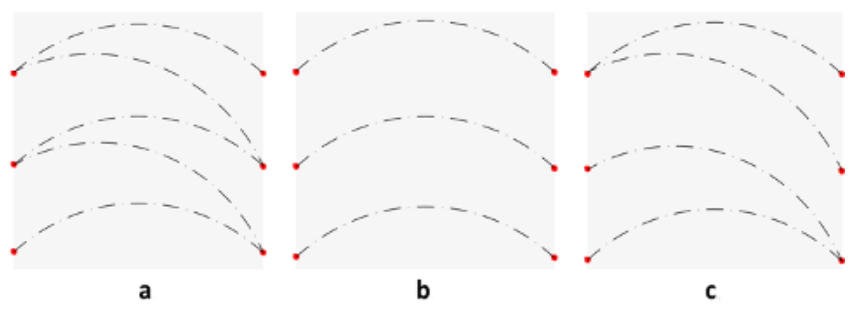

Figure 3: Curved folding strategies applied on sheet papers: a) connecting edges of two curved lines, b) arranging parallel curved lines with similar or different radius, and c) combination of both types of curved lines.

curved folding strategies. We employed a scoring and manual folding technique to create physical models for further investigation. After producing a total of 42 curved folded models by deforming papers, we have shortlisted five designs for computational procedures. Next, we have implemented a rotary table configuration $[3,48]$ to trace the contours of the mock-up models with a 3D scanner for digital design procedures. Mockup-models were digitally simulated and optimized. Finally, fabrication techniques were explored for physically re-engaging with the iterated design concepts.

\section{DESIGN PROCESS OF CURVED FOLDED MODELS}

The following three sections explicate the design process that is predicated on a feedback-oriented approach integrating physical and digital modes of design and testing. We reflect on the existing literature throughout the design process.

\subsection{Form Exploration Through Curved Folding}

The design process started with a volumetric exploration by changing the physical form of a single sheet of paper through different folding strategies (Fig.3). The primary goal was to produce initial mock-up ideas that can initiate a dialogue between the manual and digital design processes. We employed a scoring and folding 
strategy to create curved-folded forms with different formal characteristics [7]. First, we applied three folding strategies on A4 papers: a) connecting edges of two curved lines, b) arranging parallel curved lines with similar or different radius, and c) combination of both types of curved lines. Next, we repeated the same method with A3 size papers with a grammage of $150 \mathrm{~g} / \mathrm{m} 2$. Applying the aforementioned strategies on paper surfaces returned a total of 42 curved-folded models. Brief observation notes have been taken after physically forming each model to identify important design features. Physical models were individually photographed for further investigation. Documented images were classified, based on the folding method, and five models were shortlisted after a visual assessment in conjunction with the observation notes. During the evaluation and selection procedure, the key measures were the rigidity and distinctive volumetric characteristics of folded paper models. Consequently, physical models meeting these criteria from each folding strategy were included. Then, we have added the model with the lowest number of folds and the model that has the highest number of folds among all designs meeting the selection criteria. This addition aimed to examine how altering the complexity and recurrence interval of the fold lines influence 3D scanning, digital simulation, and fabrication procedures.

Initiating the design process by physical modeling facilitated the quick creation of complex non-linear surfaces. The design space was not constrained with the graphical user interface (GUI) of a computational design software [16]. Therefore, unexpected effects, problems, and potentials occurring in the physical platform gave direction to the design process [52]. Exploring design ideas through paper mock-ups provides a sense of materiality that may not be obtained through computerized models in the initial design stages. According to Groth [14], physical engagement forms a link between the mind and the material. Physical engagement with the paper provided tangible feedback regarding the constraints and the applicability of complex curved geometries. Furthermore, testing different paper sizes assisted comprehension of the curved surfaces in architectural scales. Pallasma [36] states that "understanding the architectural scale implies the unconscious measuring of the object or the building with one's body and of projecting one's body scheme into the space in question." The manual model-making process allowed recognizing physical constraints of the spatial concepts from the early design stages [20]. To illustrate, we identified splits on the surface of $150 \mathrm{~g} / \mathrm{m} 2$ papers while producing curved surfaces with perpendicular junctions between their curved reference lines. Tears that occurred on paper surfaces provided initial feedback indicating that folded geometries with steep intersections cannot be built without stretching the surface. Consequently, paper models that did not deform during the folding process were picked out for further investigation in the digital platform. For students and practicing architects, producing physical models and testing initial design ideas with digital tools can help with the assessment of complex "spatial, aesthetic and programmatic" solutions while developing initial concepts $[1,13]$. In later design stages, physical models provide limited information regarding the architectural performance of curved folded surfaces. The design process may become reliant on computational simulations to extract the technical data for testing tangible prototypes in the physical space.
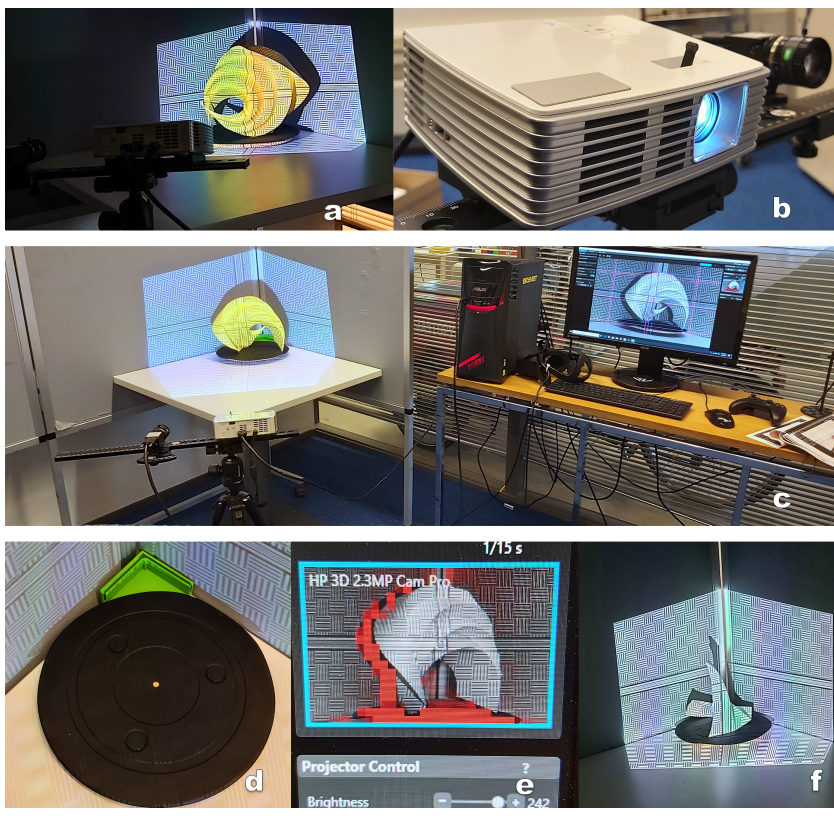

Figure 4: a) 3D scanning test in a dark environment, b) highresolution camera and projector setup, c) 3D scanning test in a well-lit environment, $d$ ) the volumetric $3 \mathrm{D}$ scanning process with the implementation of a rotary table enables 360 degrees tracing of the models, e) initial outcomes from the scanning test are displayed on the computer screen in realtime, $f$ ) with the projected grid, the structured-light scanner generates high-definition 3D models without requiring extensive optimization.

\subsection{D Scanning as a Digital Translation Method}

After finalizing the physical modeling process, our next step was switching from physical to a computational workflow. The 3D scanning method has been implemented to integrate physical and digital processes by tracing the physical data into the 3D modeling software. First, we explored scanning a comparatively simple paper model with a Kinect ${ }^{1}$ sensor. The sensor captures the volume of the physical model as point cloud data. Due to the low-resolution image capturing technology (640x480 pixels at $30 \mathrm{fps})$, the scanned model requires significant rebuilding. Undetected geometries and deformities on the surfaces make the model impractical. Running structural simulations on meshes created from point clouds can be a challenging task due to the high point count of such models. Conversely, it is possible to quickly produce higher resolution physical data with the recent advances in scanning technologies.

Next, to test the scanning capability of more recent technology, we have used an HP 3D Structured-Light Scanner that can generate high-definition 3D models without requiring extensive optimization (Fig.4). A rotary table configuration was adopted to achieve precise results in a short amount of time. The rotary table allowed 360 degrees tracing while the high-precision camera (max 0.05 percent

$\overline{{ }^{1} \text { https://support.xbox.com/sv-SE/xbox360/accessories/kinect-sensor-components }}$ 
of the object size - up to $0.05 \mathrm{~mm}$ ) captured forms in a coordinated manner with the projected 3D background grid. By using the projected grid, a clear separation between the background and the physical model was accomplished, which consequently resulted in digital models with sharp edge details. The rotary scanning setting and the high-definition scanning tool produced compact surfaces in OBJ, a geometry definition format. Thus, it was a manageable task to optimize the scanned surfaces in the digital design platform.

Employing a 3D scanning technique supported a free-form ideation process that is not constricted with computer-generated geometries. Kus et al. [26] point out that the ability to integrate 3D scan data with 3D software and virtual technologies can provide users in design and manufacture education with highly accurate data to construct complex organic shapes. For novices, computing and re-creating physically produced non-linear forms can be complicated and time-consuming. 3D scanning technology enables the exploration of ideas promptly in the physical space without using digital tools. Physically created forms can then be measured and translated into digital design software.

Linking paper mock-ups with digital tools facilitated a feedback platform in which physical data can be fed and tested digitally or the other way around. As Piper et al. [37] state, "the use of scanners to input physical geometry in real-time offers an alternative vision for computer interaction where the user is free to use any object, material or form to interface with the computer." Therefore, interactions with the physical model were not bound to a single interface or a material. Through this design process, we have observed that scanning technologies enriched the design exploration by expanding material and manipulation possibilities. Åkesson and Mueller [2] argue that "an improved human-computer interaction can potentially improve the user understanding of the structural behavior of a model, and encourage further design exploration." We believe that a fluid integration of scanning technologies can enhance initial design processes by supporting designers to use their manual skills and material knowledge while benefiting from digital design tools.

\subsection{Parametric Optimization Process}

In the next design stage, 3D geometries obtained from the scanning process were fed into the parametric design software for digital iterations. We adopted Grasshopper ${ }^{2}$ for the parametric workflow and Karamba $3 \mathrm{D}^{3}$ for digital simulations, which provided a visual programming environment as plug-ins of Rhinoceros $3 \mathrm{D}^{4}$ modeling software.The simulation algorithm transforms the mesh into a shell component to perform the analysis and assumes that it is an assembly of shells connected with elastic hinges. This process simplifies the modeling of curved folds by assuming "perfect hinges with a torsional spring related to the fold angle." [28] Such modeling allows finite element computations and it is commonly used in mechanical engineering. One of the distinctive advantages of the parametric design environment is the ability to simulate real-world applications of initial ideas created by simple mock-up models. Although the physical properties of a 1:1 scale material are not considered during the paper folding process, a material can be assigned through

\footnotetext{
${ }^{2}$ https://www.grasshopper3d.com/

${ }^{3}$ https://www.karamba3d.com/

${ }^{4}$ https://www.rhino3d.com/
}

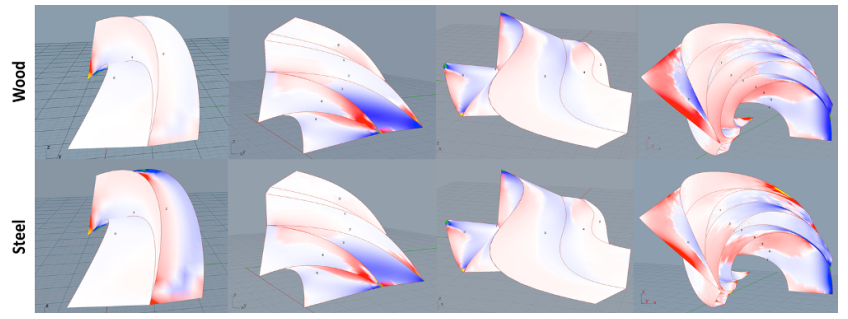

Figure 5: The digital simulation process enables a reengagement with physical models by feeding the information back into the physical realm and allows testing of the digital findings with physical prototypes. Visualizing how non-linear designs perform with different materials helped us to assess the feasibility of initial design ideas. Based on the simulations, unstable surfaces (darker red-blue gradient) have been modified or excluded for the final fabrication procedure.

computer simulation. After transferring the scanned files into the simulation algorithm, we assigned preset materials (e.g., wood, steel, etc.) with $1 \mathrm{~mm}$ thickness and loads (gravitational pull and material weight) to the model (Fig.5). Visualizing how non-linear designs perform with different materials helped us to assess the feasibility of initial design ideas. The algorithm approximates problematic areas of the curved geometry and displays with gradient blue and red colors. The gradient visualization indicates a strain caused in positive and negative directions with the flow of physical forces. During the simulation process, we observed that visually complex paper models turn out more structurally unstable areas with the increased number of curved creases. Extracting this information offered a source to apprehend the architectural, spatial and aesthetic qualities of created paper models.

Performing a digital simulation can be efficient in terms of time, cost, and material resources, and physical testing with 1:1 scale materials may not be practical during the initial design stages. Kotnik points out that [25], digital experiments need to be verified "with the performance that can be observed in reality, and a continual adaptation of the mathematical description that is driven by this feedback." Observations obtained from the parametric workflow confirm that visual programming interfaces can restrict further design exploration. Therefore, merely depending on the computational simulation can be misguiding for the design intent and planning [16]. At the same time, digital models can encourage further physical exploration by providing data for digital fabrication processes. With the digital representations of physical models, $\mathrm{CAD}$ files for subtractive fabrication processes (e.g., laser cutting, CNC milling, etc.) or 3D models for additive manufacturing (e.g., $3 \mathrm{D}$ printing) can be generated [33]. The digital simulation process enables a re-engagement with physical models by feeding the information back into the physical realm and allows testing of the digital findings with physical prototypes. Piper et al. [37] argue that digital simulations alone do not provide a tangible three-dimensional analysis that is afforded by physical models. Accordingly, comparing computational outcomes with physical outcomes by re-engaging with physical models plays a crucial role in design development. 


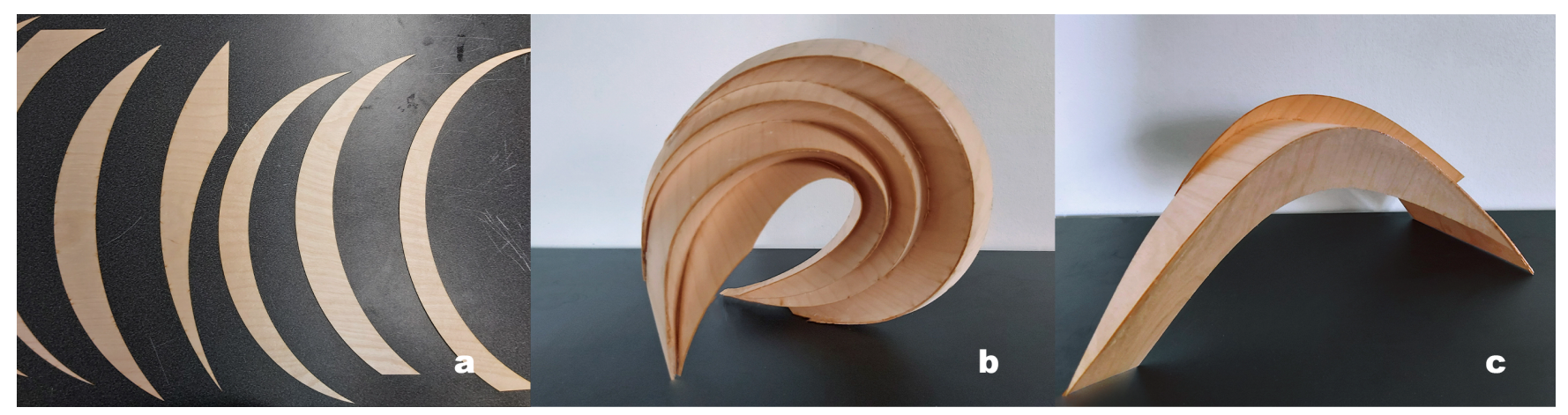

Figure 6: Various stages of assembling the curved folded plywood prototypes by integrating physical and digital design techniques: a) unassembled laser-cut components of the prototype (b) with thinner and more curved folds, c) assembled plywood prototype with larger and fewer curved folds.

\subsection{Re-Engaging with Physical Models}

Digital fabrication tools support switching from digital to physical design workflows. The laser cutting technique was explored to bring back physical engagement and test optimized digital concepts with tangible prototypes. For this process, we focused on two samples that have the highest and lowest number of curved folds among the five shortlisted designs (Fig.6). The objective was to identify differences between two iterated models in varying numbers of fold lines during the fabrication and assembly procedures.

The feedback received from previous design stages showed that the pliability of the material impacts the stability of curved folded surfaces. Accordingly, $1 \mathrm{~mm}$ thick flexible plywood sheets were utilized for laser cutting of the curved folded forms. Plywood is a versatile material that can be manually bent by applying heat on its surface to achieve desired shapes. After the digital simulation and optimization procedures, folded digital 3D models were flattened in Rhino 3D and converted to CAD (vector) drawings for the fabrication. Model surfaces were separately arranged on the $\mathrm{CAD}$ environment to implement an assembly logic suitable for the plywood bending technique. Due to the difference in material characteristics of paper and wood, fabricating with plywood led to new material constraints. Curved folded pieces were arranged on CAD by considering the direction of the wood grains to obtain the best bending performance. Digital data was fed into the laser cutter, and physical curved surfaces were extracted from $(30 \times 60 \mathrm{~cm})$ plywood sheets. During the assembly procedure, we used a heat gun 5 (Bosch GHG 23-66, 650 ${ }^{\circ} \mathrm{C} \max$ ) on the surface areas to gain more flexibility. Heat application loosens the rigid material surface and improves physical manipulations. Bent curved creases were connected from their edges with a polyurethane-based wood adhesive. Assembled models were exposed to heat again for curved creases to interact with each other while reaching the intended shape. The physical assembly process uncovered that building fewer components with larger surface areas can be as challenging as modeling complex curved folds. Attaining stable geometries with larger and less curved folds required significant heat application and physical support. In contrast, thinner and more curved folds were easier to manage and manipulate into organic forms. Increasing the number

\footnotetext{
${ }^{5}$ https://www.bosch-professional.com/kw/en/products/ghg-23-66-06012A6370
}

of creases created a dynamic interaction between curved folds and resulted in a uniform and abstract concept enabling a generative potential in various areas of application (i.e., lightweight multipurpose constructs, interior elements, building facades, etc.) As a tangible outcome of the feedback-oriented approach, two research prototypes were fabricated by shifting between physical and digital processes.

\section{DISCUSSION}

\subsection{From Paper Mock-ups to Complex Designs}

Manual paper folding tests have shown that employing mock-ups leads to complex design ideas without requiring extensive digital software knowledge. Implementing 3D scanners with a rotary table setting supports the rapid digital translation of physical models, and the parametric design platform refines hand-crafted geometries. As a tangible outcome of this conversion, initial design ideas have been manifested in physical form through laser cutting and assembly techniques. Digital and scaled physical models alone can only embody limited information [13]. Hence, following the feedbackoriented approach allowed integrating manual design skills while enriching the process via digital design tools. Applying a similar design workflow could assist processes that require creative architectural solutions [52] (i.e., massing, form-finding, etc.).

Using paper as a medium to explore complex curved folded surfaces forms a tangible design setting beyond traditional mouse and keyboard-based interactions [19]. According to Piper et al. [37], "most three-dimensional renderings and simulations are still viewed on the computer screen, which as a two-dimensional and visual form of representation, does not support a more intuitive threedimensional analysis that is afforded by physical models." Initiating the design process with simple mock-ups provides a sense of materiality by freeing exploration from two-dimensional digital constraints. Although digital tools can generate complex curved models, they do not afford direct hands-on control and quick manipulation of design ideas. Physical explorations delivered unintentional effects and solutions that directed the progression of design ideas. For instance, combining both types of curved lines, increasing the recurrence interval, and applying physical bending created organic 
twisting volumes with spatial qualities. Using the same folding strategy with fewer curved folded lines delivered freestanding designs establishing a relationship with the floor area. Through manual scoring and folding on paper, it was possible to create, systematize, and simultaneously test curved folding strategies that may otherwise be overlooked in the digital platform due to a vast quantity of options.

\subsection{Integrating Physical and Digital}

This paper anatomized the process of switching between physical and digital modes of design for creating a specific set of complex curved geometries. We explored existing conventions in architecture to understand how physical and digital design techniques can facilitate a feedback-oriented design approach. The physical-digital transition has been previously studied through tangible approaches to support creativity (i.e., [3, 19, 37, 41, 48]). Our research process can open up new trajectories for tangible user interfaces, interactive tabletops, and creativity support tools within the HCI research community. For example, converting simple ideas into digital models in real-time, similar to the physical proxy method of Sheng et al. [41], is an exciting area for further research through a feedbackoriented design approach with a focus on planar surfaces. Another potential application area is, adopting the feedback-oriented approach to develop seamless fabrication methods or settings (i.e., the bi-directional fabrication method of Weichel et al. [48]) for bridging the gap between physical and digital modes of design. Furthermore, exploring tactile manipulation of scanned paper models with immersive technologies such as mixed reality (MR) and virtual reality (VR) $[4,43,51]$ in the context of tabletop systems [17] could enable intuitive $3 \mathrm{D}$ explorations. Besides paper, there is also room for testing different mock-up modeling materials (such as clay, sponge, textiles, etc.) to expand exploration alternatives and promote creative solutions for various programmatic requirements.

\subsection{Limitations}

While producing conceptual mock-ups, we encountered some limitations of the paper as an exploratory material. Due to the rectangular form factor, some folding strategies delivered developable surfaces with sharp edges that may not be suitable for structural applications. When rotating or twisting the paper surfaces, physical deformation occurring on the curved folds may also impact the design process. Furthermore, during the 3D scanning process, we identified some technical limitations that may prevent capturing physical data efficiently. 3D scanning processes rely on the visual feedback obtained from a physical model through cameras and sensors. Thus, the physical model may need to be sufficiently illuminated for achieving the optimal 3D results.

Besides laser cutting, we explored 3D printing, an additive fabrication method. However, the 3D printing process lacked material engagement, consumed an extensive amount of time, and did not offer diverse material options that can be physically manipulated. Therefore, we mainly focused on the laser cutting process, which was more suitable for achieving material engagement alongside complex curved folded models.

\section{CONCLUSION AND FUTURE WORK}

This paper presented a feedback-oriented design process employing the curved folding technique as a test case to combine physical and digital workflows of architectural design. The transitional approach demonstrated in this work contributes to supporting intuitive non-linear form explorations in the initial design processes through physical-digital integration. Documentation of this design process provides insights and unlocks new territories to explore similar design approaches in the HCI domain. Complex kinematics of curved folded surfaces offer many potentials for future research by challenging the contemporary digital design and fabrication technologies. For expanding our research, we will conduct contextual interviews with architects and acquire an in-depth understanding of the current ideation processes as well as the role of physical and digital tools[15]. We plan to apply the learnings of these interviews to develop our design approach.

\section{REFERENCES}

[1] Asterios Agkathidis. 2015. Generative Design : Form-Finding Techniques in Architecture. Laurence King Publishing Ltd, London United Kingdom. https: //doi.org/10.1192/bjp.111.479.1009-a

[2] D Åkesson and Caitlin Mueller. 2018. Using 3D direct manipulation for real-time structural design exploration. Computer-Aided Design and Applications 15 (2018), 1-10. https://doi.org/10.1080/16864360.2017.1355087

[3] D Anderson, J L Frankel, J Marks, A Agarwala, P Beardsley, J Hodgins, D Leigh, K Ryall, E Sullivan, and J S Yedidia. 2000. Tangible interaction + graphical interpretation: A new approach to 3D modeling. In Proceedings of the ACM SIGGRAPH Conference on Computer Graphics. ACM, New York, 15.

[4] Ryan Arisandi, Mai Otsuki, Asako Kimura, Fumihisa Shibata, and Hideyuki Tamura. 2014. Virtual handcrafting: Building virtual wood models using tooldevice. Proc. IEEE 1 (2014), 10. https://doi.org/10.1109/JPROC.2013.2294243

[5] Ravin Balakrishnan, George Fitzmaurice, Gordon Kurtenbach, and Karan Singh. 1999. Exploring Interactive Curve and Surface Manipulation Using a Bend and Twist Sensitive Input Strip. In Proceedings of the 1999 Symposium on Interactive 3D Graphics (Atlanta, Georgia, USA) (I3D '99). Association for Computing Machinery, New York, NY, USA, 111-118. https://doi.org/10.1145/300523.300536

[6] Shajay Bhooshan, Vishu Bhooshan, Ashwin Shah, Henry Louth, and David Reeves. 2015. Curve-folded form-work for cast, compressive skeletons. In Simulation Series. ACM, New York, 6.

[7] Shajay Bhooshan, Mustafa El-Sayed, and Suryansh Chandra. 2014. Designfriendly strategies for computational form-finding of curved-folded geometries: A case study. In Simulation Series. 9781632662231, New York, 8.

[8] Paulo Blikstein and Dennis Krannich. 2013. The makers' movement and FabLabs in education. In IDC '13: Proceedings of the 12th International Conference on Interaction Design and Children. Association for Computing Machinery, New York, NY, USA, 613-616. https://doi.org/10.1145/2485760.2485884

[9] Suryansh Chandra, Axel Körner, Antiopi Koronaki, Rachelle Spiteri, Radhika Amin, Samidha Kowli, and Michael Weinstock. 2015. Computing curved-folded tessellations through straight-folding approximation. In Simulation Series. Society for Computer Simulation International, San Diego, CA, USA, 152-159.

[10] Nick Dunn. 2012. Digital Fabrication in Architecture. Computer 1 (2012), 192. https://doi.org/10.1109/MC.2012.407

[11] Sean Follmer, David Carr, Emily Lovell, and Hiroshi Ishii. 2010. CopyCAD: Remixing Physical Objects with Copy and Paste from the Real World. In Adjunct Proceedings of the 23nd Annual ACM Symposium on User Interface Software and Technology (New York, New York, USA) (UIST '10). Association for Computing Machinery, New York, NY, USA, 381-382. https://doi.org/10.1145/1866218.1866230

[12] Christopher Frayling. 1993. Research in Art and Design. Royal College of Art Research Papers 1 (1993), 6.

[13] Gramazio Fabio and Matthias Kohler. June 2014. Made by Robots: Challenging Architecture at a Larger Scale. Fohn Wiley \& Sons, Incorporated, 2014. Made by Ro (June 2014), 136.

[14] Groth Camilla. 2017. Making Sense Through Hands: Design and Craft Practice Analysed as Embodied Cognition. Ph.D. Dissertation. Aalto University.

[15] Emrecan Gulay. 2020. Merging Physical and Digital in the Initial Design Stages of Architecture. In Companion Proceedings of the 2020 Conference on Interactive Surfaces and Spaces (Virtual Event, Portugal) (ISS '20). Association for Computing Machinery, New York, NY, USA, 101-105. https://doi.org/10.1145/3380867.3427409

[16] Emrecan Gulay and Andrés Lucero. 2019. Integrated Workflows: Generating Feedback Between Digital and Physical Realms. In In Proceedings of the 2019 CHI Conference on Human; Paper 60. ACM, ACM, New York, NY, USA, 15 pages. 
https://doi.org/10.1145/3290605.3300290

[17] Björn Hartmann, Meredith Ringel Morris, Hrvoje Benko, and Andrew D Wilson. 2010. Pictionaire: Supporting collaborative design work by integrating physical and digital artifacts. In Proceedings of the ACM Conference on Computer Supported Cooperative Work, CSCW. Association for Computing Machinery, New York, NY, USA, 421-424. https://doi.org/10.1145/1718918.1718989

[18] David A Huffman. 1976. Curvature and Creases: A Primer on Paper. IEEE Trans. Comput. 25, 10 (1976), 1010-1019. https://doi.org/10.1109/TC.1976.1674542

[19] Hiroshi Ishii, Carlo Ratti, Ben Piper, Yao Wang, Assaf Biderman, and Eran BenJoseph. 2004. Bringing clay and sand into digital design - continous tangible user interfaces. BT Technology fournal 22, 4 (2004), 287-299. https://doi.org/10.1023/B BTTJ.0000047607.16164.16

[20] Lisa Iwamoto. 2013. Digital Fabrications (1st ed. ed.). Princeton Architectural Press, New York, NY.

[21] Martin Kilian, Simon Flöry, Zhonggui Chen, Niloy J Mitra, Alla Sheffer, and Helmut Pottmann. 2008. Curved folding. ACM Transactions on Graphics 27, 3 (2008), 1-9. https://doi.org/10.1145/1360612.1360674

[22] Jan Knippers and Thomas Speck. 2012. Design and construction principles in nature and architecture. Bioinspiration and Biomimetics 7 (2012), 1-10. https //doi.org/10.1088/1748-3182/7/1/015002

[23] Richard Duks Koschitz. 2014. Computational design with curved creases : David Huffman's approach to paperfolding. Ph.D. Dissertation. Massachusetts Institute of Technology. http://hdl.handle.net/1721.1/93013

[24] Ilpo Koskinen, John Zimmerman, Thomas Binder, Johan Redstrom, and Stephan Wensveen. 2011. Design Things: Models, Scenarios, Prototypes. In Design research through practice: From the Lab, Field, and Showroom. Morgan Kaufmann, Boston https://doi.org/10.1016/B978-0-12-385502-2.00008-0

[25] Toni Kotnik. 2011. Das Experiment als Entwurfsmethode Zur Möglichkeit der Integration naturwissenschaftlichen Arbeitens in die Architektur. , in Moravansky,A. and Kirchengast, $A$ Experiment (2011), $24-53$.

[26] A Kus, E Unver, and A Taylor. 2009. A comparative study of 3D scanning in engineering, product and transport design and fashion design education Computer Applications in Engineering Education 17, 3 (2009), 263-271. https: //doi.org/10.1002/cae.20213

[27] R Laing, M Leon, J Isaacs, and D Georgiev. 2015. Scan to BIM: the development of a clear workflow for the incorporation of point clouds within a BIM environment In Building Information Modelling (BIM) in Design, Construction and Operations. WIT Press, Barcelona, Spain, 279 - 289. https://doi.org/10.2495/bim150241

[28] Arthur Lebée. 2015. From folds to structures, a review. https://doi.org/10.1260/ 0266-3511.30.2.55

[29] Ting Uei Lee, Zhong You, and Joseph M Gattas. 2018. Elastica surface generation of curved-crease origami. International fournal of Solids and Structures 136-137 (2018), 13-27. https://doi.org/10.1016/j.ijsolstr.2017.11.029

[30] Daniel Leithinger and Hiroshi Ishii. 2010. Relief: A scalable actuated shape display. In TEI'10 - Proceedings of the 4th International Conference on Tangible Embedded, and Embodied Interaction. Association for Computing Machinery, New York, NY, USA, 221-222. https://doi.org/10.1145/1709886.1709928

[31] Daniel Leithinger, Dávid Lakatos, Anthony Devincenzi, and Matthew Blackshaw. 2011. Recompose -Direct and gestural interaction with an actuated surface. In ACM SIGGRAPH 2011 Emerging Technologies, SIGGRAPH'11. Association for Computing Machinery, New York, NY, USA, Article 13, 1 pages. https://doi.org $10.1145 / 2048259.2048272$

[32] Gregg Lynn. 1993. Folding in Architecture. John Wiley Sons Ltd, New York. 28-47 pages. https://doi.org/10.1002/9781118795811.ch2

[33] Scott Marble. 2013. Digital Workflows in Architecture. Birkhäuser, Berlin, Basel. https://doi.org/10.1515/9783034612173

[34] Ryuma Niiyama and Yoichiro Kawaguchi. 2008. Gemotion Screen: A Generative, Emotional, Interactive 3D Display. In ASIAGRAPH 2008 PROCEEDINGS. ACM, New York, USA, 6.

[35] Kas Oosterhuis, Henriette Bier, Cas Aalbers, and Sander Boer. 0. File to Factory and Real Time Behavior in ONL-Architecture. In Proceedings of the 23rd Annual Conference of the Association for Computer Aided Design in Architecture and the 2004 Conference of the AIA Technology in Architectural Practice Knowledge Community. Institutional Repository, Cambridge, 12. http://resolver.tudelft.nl/ uuid:01f55888-8034-42ec-b8e5-c9b4afeaaae7

[36] Juhani Pallasmaa. 2006. Eyes of the skin: Architecture and the senses. Architect 1 (2006), 128. https://www.wiley.com/en-us/The+Eyes+of+the+Skin\%3A+ Architecture+and+the+Senses\%2C+3rd+Edition-p- 9781119941286
[37] Ben Piper, Carlo Ratti, and Hiroshi Ishii. 2002. Illuminating clay: A 3-D tangible interface for landscape analysis. In Conference on Human Factors in Computing Systems - Proceedings. Association for Computing Machinery, New York, NY, USA, 355-362.

[38] Kåre Stokholm Poulsgaard and Lambros Malafouris. 2017. Models, mathematics and materials in digital architecture. In Cognition beyond the Brain: Computation, Interactivity and Human Artifice, Second Edition. Springer, Cham, USA. https: //doi.org/10.1007/978-3-319-49115-8\{ \}14

[39] Majken K Rasmussen, Esben W Pedersen, Marianne G Petersen, and Kasper Hornbæk. 2012. Shape-changing interfaces: A review of the design space and open research questions. In Conference on Human Factors in Computing Systems Proceedings. Association for Computing Machinery, New York, NY, USA, 735-744. https://doi.org/10.1145/2207676.2207781

[40] Nenad Sekularac, Jelena Ivanovic-Sekularac, and Jasna Cikic-Tovarovic. 2012. Folded structures in modern architecture. Facta universitatis - series: Architecture and Civil Engineering 1 (2012), 15. https://doi.org/10.2298/fuace1201001s

[41] Jia Sheng, Ravin Balakrishnan, and Karan Singh. 2006. An interface for virtual 3D sculpting via physical proxy. Proceedings - GRAPHITE 2006: 4th International Conference on Computer Graphics and Interactive Techniques in Australasia and Southeast Asia GRAPHITE '06 (2006), 213-220. https://doi.org/10.1145/1174429. 1174467

[42] Michael Siebenbrodt and Lutz Schöbe. 2009. Bauhaus. Parkstone International, New York.

[43] Keng Hua Sing and Wei Xie. 2016. Garden: A mixed reality experience combining virtual reality and 3D reconstruction. In Conference on Human Factors in Computing Systems - Proceedings. Association for Computing Machinery, New York, NY, USA, 180-183. https://doi.org/10.1145/2851581.2890370

[44] Hyunyoung Song, Francois Guimbretiere, and Hod Lipson. 2009. The ModelCraft Framework: Capturing Freehand Annotations and Edits to Facilitate the 3D Model Design Process Using a Digital Pen. ACM Trans. Comput.-Hum. Interact. 16, 3, Article 14 (Sept. 2009), 33 pages. https://doi.org/10.1145/1592440.1592443

[45] Kostas Terzidis. 2006. Algorithmic architecture. Architectural Press, USA. https: //doi.org/10.4324/9780080461298

[46] Yingmo Wang, Zexiao Li, Zhuang Fu, Fengzhou Fang, and Xiaodong Zhang. 2019. Radial scan form measurement for freeform surfaces with a large curvature using stylus profilometry. Measurement Science and Technology 30, 4 (2019), 045010. https://doi.org/10.1088/1361-6501/ab052f

[47] Christian Weichel, Jason Alexander, Abhijit Karnik, and Hans Gellersen. 2015. SPATA: Spatio-Tangible Tools for Fabrication-Aware Design. In Proceedings of the Ninth International Conference on Tangible, Embedded, and Embodied Interaction (Stanford, California, USA) (TEI '15). Association for Computing Machinery, New York, NY, USA, 189-196. https://doi.org/10.1145/2677199.2680576

[48] Christian Weichel, John Hardy, Jason Alexander, and Hans Gellersen. 2015. ReForm: Integrating physical and digital design through bidirectional fabrication. In UIST 2015 - Proceedings of the 28th Annual ACM Symposium on User Interface Software and Technology. Association for Computing Machinery, New York, NY, USA, 93-102. https://doi.org/10.1145/2807442.2807451

[49] Michael Wessely, Theophanis Tsandilas, and Wendy E. Mackay. 2018. ShapeAware Material: Interactive Fabrication with ShapeMe. In Proceedings of the 31st Annual ACM Symposium on User Interface Software and Technology (Berlin, Germany) (UIST '18). Association for Computing Machinery, New York, NY, USA, 127-139. https://doi.org/10.1145/3242587.3242619

[50] Karl D.D. Willis, Cheng Xu, Kuan-Ju Wu, Golan Levin, and Mark D. Gross. 2010. Interactive Fabrication: New Interfaces for Digital Fabrication. In Proceedings of the Fifth International Conference on Tangible, Embedded, and Embodied Interaction (Funchal, Portugal) (TEI '11). Association for Computing Machinery, New York, NY, USA, 69-72. https://doi.org/10.1145/1935701.1935716

[51] Takuya Yamamoto, Maho Kawagoe, Mai Otsuki, Fumihisa Shibata, and Asako Kimura. 2017. Enjoyable carving with ChiselDevice in mixed reality space. In Proceedings of the ACM Symposium on Virtual Reality Software and Technology, VRST. Association for Computing Machinery, New York, NY, USA, 2. https: //doi.org/10.1145/3139131.3143419

[52] Albena Yaneva. 2005. Scaling up and down: Extraction trials in architectural design. https://doi.org/10.1177/0306312705053053

[53] John Zimmerman, Jodi Forlizzi, and Shelley Evenson. 2007. Research through design as a method for interaction design research in HCI. In Conference on Human Factors in Computing Systems - Proceedings. Association for Computing Machinery, New York, NY, USA, 493-502. https://doi.org/10.1145/1240624.1240704 